\title{
Controlador robusto basado en la técnica QFT para convertidores DC-DC buck - boost como regulador de voltaje en generadores fotovoltaicos
}

\section{Robust controller based on the technical QFT for converters DC-DC buck - boost as photovoltaic generator voltage regulator}

\author{
Rafael Augusto Núñez-Rodriguez ${ }^{1}$, Omar Pinzón-Ardila ${ }^{2}$ \\ ${ }^{1}$ Grupo de investigación en Control Industrial, Facultad de Ingeniería Electrónica., Universidad Pontificia Bolivariana, \\ Colombia. Email: ing.rafaeln@gmail.com \\ ${ }^{2}$ Grupo de investigación en Control Industrial, Facultad de Ingeniería Electrónica., Universidad Pontificia Bolivariana, \\ Colombia. Email: omar.pinzon@upb.edu.co
}

RECIBIDO: Marzo 17, 2017. ACEPTADO: Agosto 07, 2017. Versión FinAL: Agosto 17, 2017

\begin{abstract}
RESUMEN
En este artículo se presenta un controlador robusto basado en la teoría de realimentación cuantitativa, QFT de su sigla en inglés, para un convertidor DC-DC Buck-Boost en modo de conducción continua que regula el voltaje suministrado por un generador fotovoltaico PV-UD190HA6 de $190 \mathrm{Wp}$. La estrategia de control propuesta se diseña utilizando técnicas de diseño basado en modelo, utilizando el entorno de Simulink de Matlab® y la Toolbox Simscape para el modelamiento de sistemas físicos. El esquema de control QFT se ha modificado a partir de una estructura tipo Predictor de Smith para sistemas de fase no mínima, donde se muestra un comportamiento robusto del voltaje de salida del regulador ante cambios en la impedancia de carga, variación del punto de operación del convertidor como consecuencia de la variación de irradiación y temperatura del generador fotovoltaico, consiguiendo que el controlador propuesto cumpla con las especificaciones de desempeño establecidas y reduciendo el esfuerzo en la señal de control.
\end{abstract}

PALABRAS CLAVE: Control robusto QFT; convertidor DC-DC buck-boost; generador fotovoltaico.

\begin{abstract}
In this paper we present a robust controller based on the quantitative feedback theory (QFT) for a DC-DC converter Buck-Boost in continuous conduction mode that regulates the voltage supplied by a PV-UD190HA6 photovoltaic generator $190 \mathrm{Wp}$. The proposed control strategy is designed using model-based design techniques using the Simlink ${ }^{\circledR}$ environment of Matlab ${ }^{\circledR}$ and the Simscape Toolbox for modeling physical systems. The QFT control scheme has been modified from a Smith Predictor type structure for non-minimal phase systems, showing a robust behavior of the regulator output voltage before changes in the load impedance, variation of the operating point of the converter as a consequence of the variation of irradiation and temperature of the photovoltaic generator, getting the proposed controller to comply with the established performance specifications and reducing the effort in the control signal.
\end{abstract}

KEYWORDS: Robust control QFT; buck-boost converter; PV generator.

ISSN Impreso: 1657 - 4583, En Línea: 2145 - 8456

Este artículo puede compartirse bajo la licencia CC BY-ND 4.0 y se referencia usando el siguiente formato: R. Núñez, O. Pinzón, "Controlador robusto basado en la técnica QFT para convertidores DC-DC buck - boost como regulador de voltaje en generadores fotovoltaicos," Rev. UIS Ing., vol. 17, no. 1, pp. 243-250, 2018. Doi: https://doi.org/10.18273/revuin.v17n1-2018024 


\section{INTRODUCCIÓN}

El punto de operación adecuado de los generadores fotovoltaicos está ligado a las condiciones ambientales, además de la eficiencia del regulador, especialmente la eficiencia con que se genera el ciclo de conmutación del convertidor para mantener la tensión de salida en un rango aceptable. Con miras a incrementar la eficiencia de los convertidores DC-DC utilizados como reguladores de voltaje se han estudiado diferentes técnicas de control, para convertidores DC-DC Buck-Boost. Técnicas de control por modelo interno con dos grados de libertad aplicado a un convertidor elevador, presentan buenos índices de desempeño para el convertidor a pesar de la restricción de ancho de banda dada su dinámica de fase no mínima [1]. De igual forma las estrategias de control robusto por modo deslizante se han implementado para convertidores tipo Boost y tipo Buck-Boost [2], [3], que han permitido conseguir robustez del sistema ante variaciones de voltaje de entrada y perturbaciones por cambios de la impedancia de carga. Por otra parte se han formulado algoritmos de control predictivo para modelos sub-óptimos con el fin de asegurar estabilidad robusta con mayor eficiencia de un convertidor Buck-Boost utilizando la función de Lyapunov para minimizar la función de costo [4], [5]. Otras estrategias para optimizar la transferencia de energía del generador fotovoltaico a la carga se han formulado con la combinación de técnicas de control clásica en combinación con algoritmos MPPT aplicados en un convertidor DC-DC Buck-Boost [6]. En este trabajo se propone un controlador robusto QFT con dos grados de libertad y una estructura basada en el Predictor de Smith modificado para sistemas de fase no mínima [7]. En la Sección 2 se plantea el modelamiento matemático del generador fotovoltaico y el convertidor Buck-Boost, seguido se muestra el diseño del controlador robusto QFT en la Sección 3. En la sección 4 se discuten los resultados del desempeño de la estrategia de control propuesta y por último en la Sección 5 se plantean las conclusiones.

\section{MODELADO}

\subsection{Generador fotovoltaico}

Se propone un modelo de simulación conformado por un generador fotovoltaico construido con cuatro módulos en serie PV-UD190HA6 de $190 \mathrm{Wp}$, conectados a un convertidor DC-DC Buck-Boost con carga resistiva que se encarga de regular el voltaje de salida entre $75 V_{D C}$ y $125 V_{D C}$. Cada módulo se modela como una unión PN que exhibe un comportamiento no lineal de la respuesta para la relación voltaje/corriente del generador.

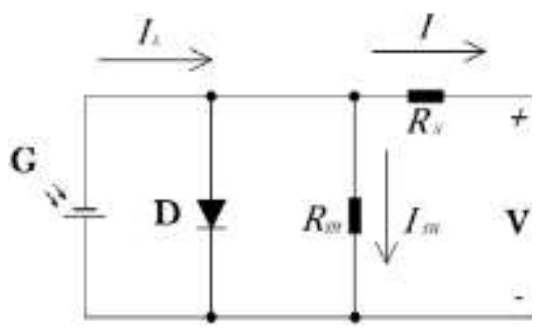

Figura 1. Circuito equivalente de generador fotovoltaico.

En la Figura 1 se muestra el circuito equivalente de una celda de un generador fotovoltaico. La corriente $I_{L}$ generada por el panel solar es directamente proporcional a la cantidad de radiación solar impresa sobre la celda, lo cual permite que se genere una corriente $I_{D}$ en el diodo $D$, conformando de esta forma la curva $V-I$ del generador[8]. $R_{S}$ corresponde a la resistencia de cada celda y $R_{S H}$ a la resistencia paralelo en condiciones ideales, la cual se puede despreciar dado su valor elevado. La corriente $I$ generada por la celda está dada por la ecuación (1).

$$
I=I_{L}-I_{o}\left(e^{\frac{q\left(V+I R_{S}\right)}{n k T}-1}\right)-\frac{V+I R_{S}}{R_{p}}
$$

Donde la fotocorriente $I_{L}$, depende de la primera y segunda temperatura de referencia y se determina por la ecuación (2).

$$
I_{L}=I_{S C_{T 1, n o m}}\left(\frac{G}{G_{N O M}}\right)+\left(\frac{I_{S C_{T_{2}}}-I_{S C_{T_{1}}}}{T_{2}-T_{1}}\right)
$$

La correinte $I_{o}$ en la ecuación (1) representa la corriente de saturación del diodo que está dada por la ecuación (3).

$$
I_{o}=\left(\frac{I_{S C_{T 1}}}{e^{\frac{q V O C}{n k T_{1}}-1}}\right)\left(\frac{T}{T_{1}}\right)^{\frac{3}{n}} e^{\frac{q V_{T_{1}}}{n k\left(\frac{1}{T^{-}}-\frac{1}{T_{1}}\right)}}
$$

Donde $I_{S C_{T 1}}$ es la corriente de cortocircuito de la celda solar a un irradiación $G_{N O M}$ de $1 \mathrm{KW} / \mathrm{m}^{2}$ y $25^{\circ} \mathrm{C} . n$ corresponde a un factor de calidad del diodo, que asume un valor de 2 para material cristalino. La constante de Boltzmann se representa por $k$ y la carga del electrón es $q[9]$.

\subsection{Convertidor DC-DC Buck-Boost}

El convertidor DC-DC Buck-Boost en modo de conducción continua se muestra en la Figura 2. El comportamiento dinámico del circuito se describe a partir de la ecuación (4) y la ecuación (5), donde $u$ es una función de la conmutación, la cual toma el valor de 1 cuando el interruptor está cerrado, y 0 cuando el interruptor está abierto, de manera que se describe el 
comportamiento dinámico en ambos modos de funcionamiento del convertidor [10].

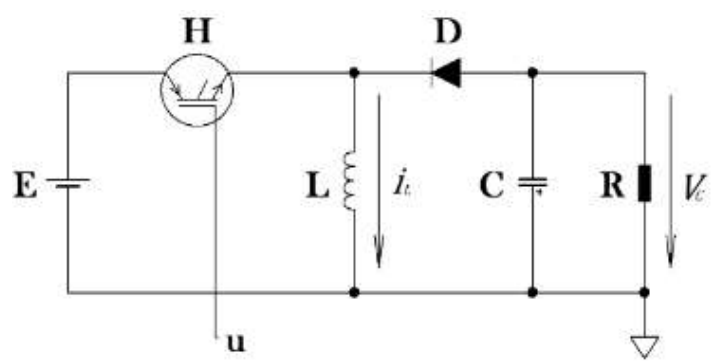

Figura 2. Convertidor DC-DC Buck-Boost en lazo abierto.

$$
\begin{gathered}
\dot{i_{L}}=\frac{1}{L}\left[E u+V c(1-u)-r i_{L}\right] \\
\dot{V} c=\frac{1}{C}\left[-i_{L}(1-u)-\frac{V c}{R}\right]
\end{gathered}
$$

Para linealizar el modelo se establece el punto de equilibrio que permite obtener un modelo en espacio de estados linealizado en un punto de operación con pequeña variación, tal como se muestra en la ecuación (6) y la ecuación (7), donde: las variables de estado se definen para el modelo promediado como $\left\langle i_{L}\right\rangle_{0}$ y $\langle V c\rangle_{0}$, el punto de equilibrio como $\alpha_{e},\left\langle V_{c}\right\rangle_{0 e}$ y $\left\langle i_{L}\right\rangle_{0 e}$, y la variación de pequeña señal como $\left.\left\langle\widetilde{V_{C}}\right\rangle_{0}, \widetilde{l_{L}}\right\rangle_{0}$ y $\tilde{\alpha}$.

$$
\begin{gathered}
\left.\dot{\overrightarrow{l_{L}}}\right\rangle_{0}=\frac{1}{L}\left(1-\alpha_{e}\right)\left\langle\widetilde{V_{C}}\right\rangle_{0}+\frac{1}{L}\left(-\left\langle V_{c}\right\rangle_{0 e}+E\right) \tilde{\alpha} \\
\left.\left\langle\dot{V_{c}}\right\rangle_{0}=-\frac{1}{C}\left(1-\alpha_{e}\right) \widetilde{l_{L}}\right\rangle_{0}-\frac{1}{R C}\left\langle\widetilde{V_{C}}\right\rangle_{0}+\frac{1}{C}\left\langle i_{L}\right\rangle_{0 e} \tilde{\alpha}
\end{gathered}
$$

La ecuación (6) y la ecuación (7), permiten calcular la función de transferencia de fase no mínima, tal como se muestra en la ecuación (8).

$$
\frac{\tilde{Y}(s)}{\tilde{\alpha}(s)}=-\frac{M^{2} E}{\alpha_{e}^{2}} * \frac{1-\tau s}{s^{2} \frac{1}{\omega_{n}^{2}}+s \frac{2 \xi}{\omega_{n}}+1}
$$

Donde la ganancia estática del convertidor $M$ se define como $M=\alpha_{e} / 1-\alpha_{e}$, la constante de tiempo $\tau$ como $\tau=$ $M^{2} L / \alpha_{e} R$, que determina la ubicación de cero en el semiplano derecho, produciendo un sistema de fase no mínima. La frecuencia natural $\omega_{n}$ y el coeficiente de amortiguamiento relativo $\xi$ se definen como $\omega_{n}=$ $\alpha_{e} / M \sqrt{1 / L C}$, y $\xi=M / 2 \alpha_{e} \sqrt{L / R^{2} C}$ respectivamente. Para definir los parámetros del convertidor se selecciona un voltaje de entrada de $100 V_{D C}$ en el punto de máxima potencia del generador y un ciclo de trabajo en el punto de equilibrio $\alpha_{e}=0.6$. Por tanto para una frecuencia de conmutación de $100 \mathrm{KHz}$ se determina una inductancia $L=500 \mathrm{uH}$, una capacitancia $C=100 \mu \mathrm{F}$ y una resistencia de carga de $R=15 \Omega$, con base en el trabajo presentado en [10].

\section{DISEÑO CONTROLADOR QFT}

\subsection{Predictor Smith para sistemas de fase no mínima}

Dadas las restricciones de ancho de banda impuestas por los sistemas de fase no mínima, se han propuesto diferentes estrategias para el diseño de controladores QFT basadas en una planta equivalente de fase mínima acoplada a un filtro pasa todo [11], no obstante tiene ciertas limitaciones en una implementación real dado que se pierde estabilidad robusta en el sistema [12]. Otro enfoque está relacionado con las limitaciones de especificaciones de desempeño con base en el corrimiento de contornos en el plano de Nichols, que implica un diseño tedioso del controlador y del pre-filtro [13]. Por otra parte, en [11] se propone una estructura fundamentada en el concepto del predictor Smith, modificado para sistemas de fase no mínima, la cual utiliza una planta estimada de fase mínima $\hat{P}_{m p}$, en un lazo interno y un filtro pasa todo $A P F$, que permite mitigar los efectos del cero en el semiplano derecho $\mathrm{S}$ y el diseño del controlador $G$ con el pre-filtro $F$, dado que es una estructura de dos grados de libertad. El esquema del controlador es como el que se muestra en la Figura 3.

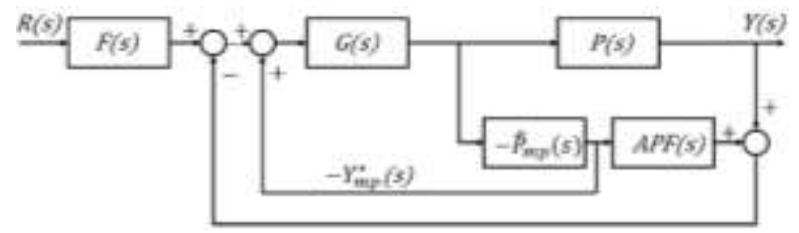

Figura 3. Esquema del controlador QFT basado en el Predictor Smith para sistemas de fase no minima.

La forma de la planta $P$ está dada por la ecuación (9), donde se observa el cero $Z=1 / a$. La planta estimada $\widehat{P}$ está dada por la ecuación (10), por tanto el sistema estimado de fase mínima está dada de acuerdo a la ecuación (11) y el filtro pasa todo por la ecuación (12).

$$
\begin{gathered}
P(s)=\frac{(-a s+1) n_{r}(s)}{d_{r}(s)} \\
\hat{P}(s)=\hat{P}_{n m p}(s)=\hat{P}_{m p}(s) A P F(s) \\
\hat{P}_{m p}(s)=\frac{(\hat{a} s+1) \hat{n}_{r}(s)}{\hat{d}_{r}(s)} \\
A P F(s)=\frac{(-\hat{a} s+1)}{(\hat{a} s+1)}
\end{gathered}
$$

Dado que se diseña un controlador robusto QFT ante variaciones paramétricas se proponen dos algoritmos que permiten seleccionar una planta adecuada $\hat{P}_{m p}$ [7]. El 
primer algoritmo busca seleccionar una planta estimada $\hat{P}_{m p}$ del espacio de incertidumbre, tal que permita que $y^{*}(s)=y(s)$ en el diagrama de la Figura 4, es decir que $|Q(s)| \leq m_{d}$ en el rango de frecuencias de interés del controlador $0 \leq \omega \leq \omega_{B W}$, donde $m_{d}$ a modo práctico se fija en $3 d B$.

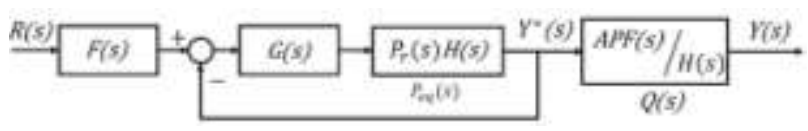

Figura 4. Esquema de Predictor Smith equivalente para sistemas de fase no minima.

Del conjunto de plantas que se seleccionaron del primer algoritmo, se toma la planta que minimiza la función de costo dada por la ecuación (13). Donde $T(s)$ representa las plantillas de la planta real y $T_{e q}(s)$ representa las plantillas de la planta equivalente. Así se define el segundo algoritmo.

$$
I_{\text {cost }}=\frac{1}{n_{\omega}} \sum_{\omega \in \Omega} \frac{A\left(T_{e q}(j \omega)\right)}{A(T(j \omega))}
$$

Con base en los algoritmos propuestos en [7] para un rango de frecuencias de interés $\omega=[1,1.7,3$, $5.5,10,30,100,300] \mathrm{Krad} / \mathrm{s}$, la planta estimada que cumple con los criterios de selección, está dada por la ecuación (14).

$$
\hat{P}_{m p}(s)=\frac{-0.06698 s-300}{6.2 \times 10^{-7} s^{2}+0.33 \times 10^{-3} s+1}
$$

\subsection{Espacio de incertidumbre}

Con base en los datos experimentales del convertidor con características similares al trabajo propuesto en [14], se puede establecer una variación en los parámetros del convertidor alrededor del $\pm 20 \%$. Para la planta nominal con una frecuencia de conmutación $F_{s}=100 \mathrm{KHz}, V_{i}=$ $100 V_{D C}$ y un punto de operación $\alpha_{e}=0.5$, una resistencia de carga $R=15 \Omega$, una inductancia $L=$ $500 \mu H$, y una capacitancia $C=100 \mu F$. Con base en la variación de los parámetros que afectan el punto de operación del convertidor se conforma un intervalo de incertidumbre. La resistencia tiene una variación tal que, $\Delta R=\left[\begin{array}{ll}12 \Omega & 18 \Omega\end{array}\right] \forall R \in \mathbb{R}$, en la inductancia $L, \Delta L=$

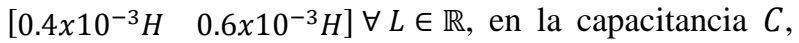
$\Delta C=\left[\begin{array}{ll}0.08 \times 10^{-3} F & 0.12 \times 10^{-3} F\end{array}\right] \forall C \in \mathbb{R} ; \quad$ lo cual permite generar una familia de plantas evaluada ante un conjunto de frecuencias de interés entre $1000 \mathrm{rad} / \mathrm{s}$ a $300 \mathrm{krad} / \mathrm{s}$, obteniendo para cada frecuencia una representación en fase $\left[{ }^{\circ}\right]$ y magnitud $[\mathrm{dB}]$ del conjunto de plantas sobre el diagrama de Nichols [15].

\subsection{Especificaciones de desempeño del controlador}

Dado que el regulador de voltaje Buck-Boost se somete a cambios de carga en la salida del convertidor, las variaciones de los parámetros en los diferentes puntos de operación, y la fluctuación aleatoria en el voltaje del generador establecido por las diferentes condiciones ambientales. En este trabajo se definen tres especificaciones de desempeño basados en la estabilidad robusta mínima recomendada donde se establece $5 \mathrm{~dB}$ en ganancia y $45^{\circ}$ en fase con un rechazo de perturbaciones de carga en el voltaje de salida del convertidor y seguimiento robusto de referencia, las cuales según la teoría de realimentación cuantitativa se definen de acuerdo a la ecuación (15), ecuación (16) y la ecuación (17) respectivamente.

$$
\begin{array}{r}
\left|\frac{y}{r}\right|=\left|\frac{L(j \omega)}{1+L(j \omega)}\right|<\delta_{u}(\omega) \\
\left|\frac{y}{d}\right|=\left|\frac{1}{1+L(j \omega)}\right|<\delta_{S}(\omega) \\
\alpha_{L}(\omega) \leq\left|\frac{P G F(j \omega)}{1+L(j \omega)}\right| \leq \alpha_{U}(\omega)
\end{array}
$$

Para resolver estas inecuaciones, se debe cuantificar los parámetros $\delta_{u}(\omega), \delta_{S}(\omega), \alpha_{L}(\omega)$ y $\alpha_{U}(\omega)$, bien sea como constantes o a partir de funciones de transferencia que representen la dinámica deseada de la planta en lazo cerrado [16]. El criterio de estabilidad robusta se define con $\delta_{u}(\omega)=1.3$, dando como resultado un margen de fase de $45^{\circ}$ y margen de ganacia $5 d B$. El rechazo de perturbaciones a la salida del convertidor, se define a partir del parámetro $\delta_{S}(\omega)$ y la ecuación (18) [17]. Éste se define como una función de transferencia que representa la dinámica deseada de la planta ante una perturbación de carga en el convertidor, por lo tanto se define un tiempo de establecimiento de $0.01 s$ de la salida ante una perturbación tipo escalón unitario, condicionado a la función de sensibilidad del sistema. Para definir la función de transferencia $\delta_{S}(\omega)$, se aplica el método de asignación de polos [16].

$$
\delta_{S}(\omega)=\frac{s^{2}+153.5 s}{s^{2}+307 s+23560}
$$

Para el seguimiento de referencias se diseña un sistema de dos grados de libertad, cuyo pre-filtro se define a partir de dos funciones de transferencia que sirven de límite superior $\alpha_{U}(\omega)$ y límite inferior $\alpha_{L}(\omega)$ de la respuesta dinámica del sistema en lazo cerrado [13]. El límite superior tiene una respuesta subamortiguada con un sobrepaso de $10 \%$ y tiempo de establecimiento $t_{s s}=$ $5 \mathrm{~ms}$, de igual forma, la respuesta sobreamortiguada obedece al mismo tiempo de establecimiento. A partir de estos parámetros se construye una función de transferencia de segundo orden con un cero que represente 
la respuesta dinámica superior deseada, tal como se muestra en la ecuación (19). Por su parte, El parámetro $\alpha_{L}(\omega)$ se ajusta a una función de transferencia con tres polos reales no repetidos, cuyo polo dominante responde al tiempo de establecimiento requerido [18]. La función de trasferencia se define en la ecuación (20).

$$
\begin{gathered}
\alpha_{U}(\omega)=\frac{676.6 s+1831000}{s^{2}+1600 s+1831000} \\
\alpha_{L}(\omega)=\frac{3965 \times 10^{6}}{s^{3}+4872 s^{2}+7692 \times 10^{3} s+3965 \times 10^{6}}
\end{gathered}
$$

\subsection{Contornos del controlador}

A partir de las especificaciones de desempeño del controlador dadas entre la ecuación (15) a la ecuación (17) y de las funciones de transferencia que representan los parámetros $\delta_{u}(\omega), \delta_{S}(\omega), \alpha_{L}(\omega)$ y $\alpha_{U}(\omega)$, se debe conseguir una $L(j \omega)$ tal que cumpla las desigualdades establecidas en las especificaciones de desempeño, donde $L(j \omega)=G(j \omega) P(j \omega)$. Así, el problema de control se centra en determinar un controlador $G(j \omega)$ único, que cumpla con todas las especificaciones de desempeño establecidas a partir de la planta con incertidumbre $P(j \omega)$ en el rango de frecuencias de interés [19]. Para resolver el problema de control, se plantean una inecuación cuadrática por cada especificación de desempeño [20] tal como se muestra en la ecuación (21) a la ecuación (24).

$$
\begin{gathered}
p^{2}\left(1-\frac{1}{\delta_{u}^{2}}\right) g^{2}+2 p \cos (\varnothing+\theta) g \geq 0 \\
p^{2} g^{2}+2 p \cos (\varnothing+\theta) g+\left(1-\frac{1}{\delta_{s}^{2}}\right) \geq 0 \\
p_{e}^{2} p_{d}^{2}\left(1-\frac{1}{\delta_{5}^{2}}\right) g^{2}+\Psi+\left(p_{e}^{2}-\frac{p_{d}^{2}}{\delta_{5}^{2}}\right) \geq 0 \\
\Psi=2 p_{e} p_{d}\left(p_{e} \cos \left(\varnothing+\theta_{d}\right)-\frac{p_{d}}{\delta_{5}^{2}} \cos \left(\varnothing+\theta_{e}\right)\right) g
\end{gathered}
$$

El sistema de ecuaciones se resuelve a partir de métodos iterativos de acuerdo al algoritmo propuesto por Chait et al [20], donde $g$ equivale a la representación polar del controlador y $p$ representa la forma polar de la planta nominal del lazo $L_{o}$. Cada raíz del sistema de inecuaciones cuadráticas representa un lugar en el diagrama de Nichols para cada par fase $\left[^{\circ}\right]$ - magnitud $[d B]$, para las $n$ frecuencias de interés y la planta nominal, donde se generan $n$ cantidad de puntos los cuales representan los contornos de cada especificación, de manera que el único controlador $g e^{j \emptyset}$ que cumple con todas las especificaciones de desempeño es aquel que consigue llevar la función del lazo $L$ sobre los interceptos de todos los contornos de cada especificación [19].

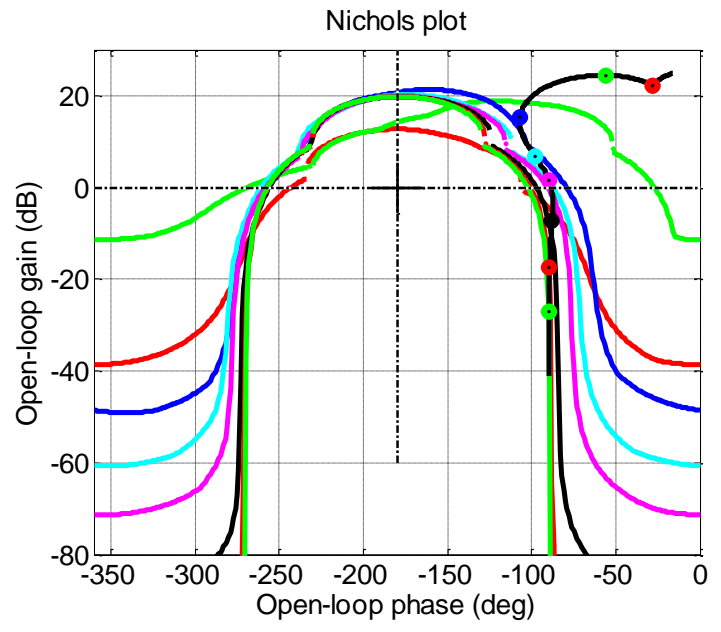

Figura 5. Loop-Shapping del controlador QFTcon $L_{0}$.

\subsection{Loop-Shapping del controlador QFT}

Mediante la técnica loop-shapping, se introduce un controlador $G(s)$ que manipule la función de lazo $L_{o}$ hasta lograr cumplir con las restricciones impuestas por las especificaciones de desempeño. La respuesta de $L_{o}$ en la frecuencia de interés debe quedar por encima del intercepto de los contornos en cada frecuencia de interés, tal como se muestre en la Figura 5. Esto se logra agregando polos y ceros a la función de lazo $L_{o}$ hasta conseguir la respuesta deseada [15]. La función de transferencia del controlador diseñado se muestra en la ecuación (25).

$$
G_{c}=\frac{0.063 s^{3}+265 s^{2}+2.3 \times 10^{5} s+2.1 \times 10^{5}}{s^{3}+9390 s^{2}+3.527 \times 10^{6} s}
$$

Dado que se propuso una estructura de dos grados de libertad capaz de hacer seguimiento de señales de referencia, es importante conformar el pre-filtro de la estructura DOF. En el diseño del pre-filtro, se tomó como referencia la solución de la inecuación cuadrática dada en la ecuación (23) y la ecuación (24), así, se debe buscar que la respuesta en frecuencia de la función $L$ se encuentre entre el límite superior y límite inferior de las funciones $\alpha_{L}(\omega)$ y $\alpha_{U}(\omega)$. La ecuación (26) permite que la respuesta en el dominio de la frecuencia del sistema se ajuste a los límites impuestos por las funciones $\alpha_{L}(\omega)$ y $\alpha_{U}(\omega)$.

$$
F=\frac{2.9 \times 10^{11}}{s^{3}+1.5 \times 10^{5} s^{2}+3.4 \times 10^{8} s+2.9 \times 10^{11}}
$$

\section{RESULTADOS}

El esquema de control robusto, se valida utilizando técnicas de diseño basado en modelo bajo el entorno de Simulink de Matlab® y con el apoyo de la Toolbox Simscape para el modelamiento de sistemas físicos. En el modelo de simulación se cuantificó la lógica propuesta 
por el control robusto para generar una señal PWM de $100 \mathrm{KHz}$ con ciclo de trabajo en función de la acción de control, donde se aplica a la puerta de un MOSFET canal $\mathrm{N}$. El controlador se implementa a partir de las funciones de transferencia en tiempo continuo. El generador fotovoltaico se modela a partir de las ecuaciones propuestas en [8] y el modelo de simulación Renewable Energy Systems documentados en la Toolbox Simscape de Mtalabß. El modelo de simulación se muestra en la Figura 6.

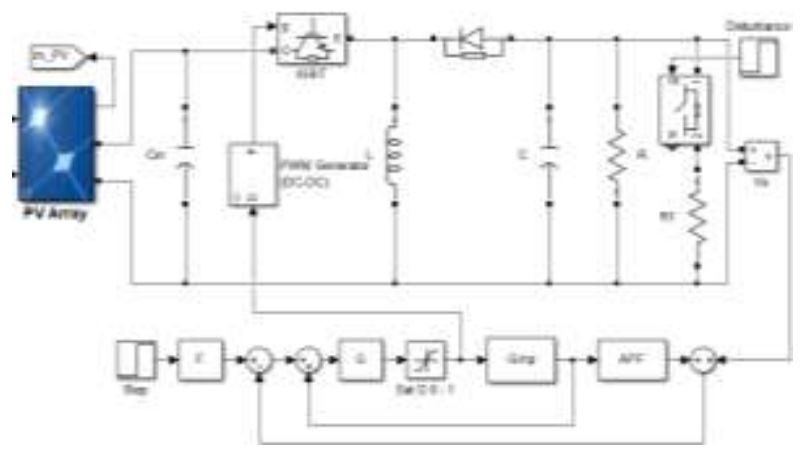

Figura 6. Modelo de simulación regulador de tensión BuckBoost.

La respuesta del convertidor se valida cuando el generador fotovoltaico de $760 \mathrm{Wp}$ genera $117 V_{D C}$ en el punto máximo de potencia y se establece una tensión de referencia en la salida de $-75 V_{D C}$. La respuesta del convertidor se muestra en la Figura 7, donde se presenta un sobrepaso cercano al 3\%, y un tiempo de establecimiento inferior a $6 \mathrm{~ms}$.

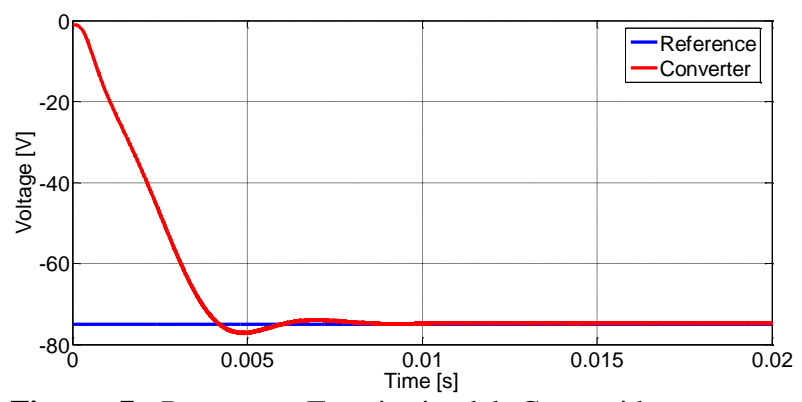

Figura 7. Respuesta Transitoria del Convertidor con una referencia de $-75 V_{D C}$.

En la Figura 8 se observa la respuesta del convertidor ante una referencia de $-125 V_{D C}$ donde se presenta un sobrepaso cercano al $8 \%$, y un tiempo de establecimiento inferior a $8 \mathrm{~ms}$, lo que implica que a pesar del cambio del punto de operación del convertidor el controlador mantiene buenos índices de desempeño.

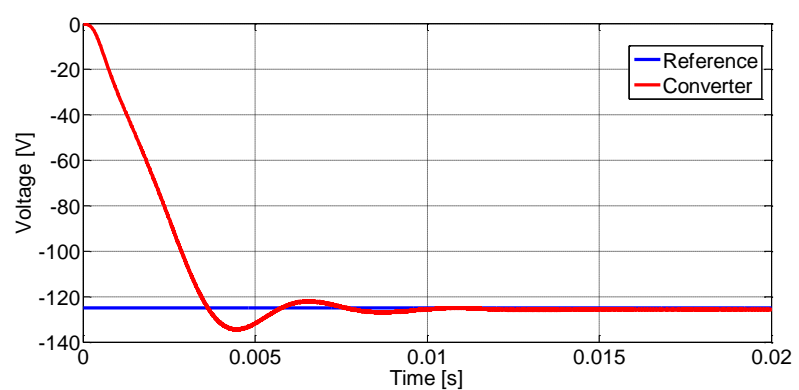

Figura 8. Respuesta Transitoria del Convertidor con una referencia de-125 $V_{D C}$.
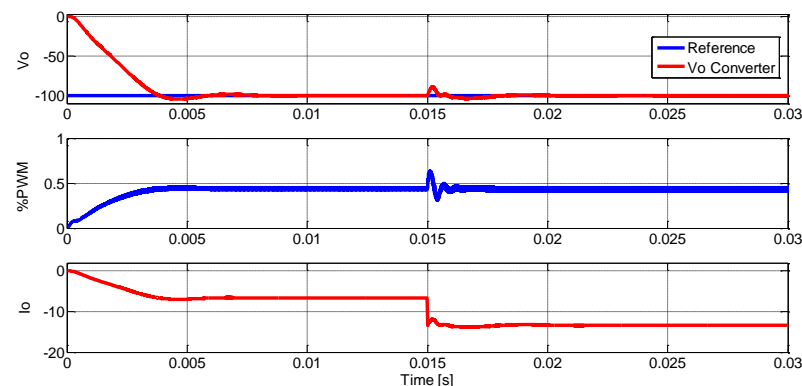

Figura 9. Respuesta transitoria ante perturbación de carga.

Dada la estabilidad del sistema en lazo cerrado, se analiza la respuesta del convertidor en presencia de una perturbación en la corriente de salida ante un cambio abrupto en la resistencia de carga del $50 \%$ a los $15 \mathrm{~ms}$ de simulación. La respuesta temporal se observa en la Figura 9, con una variación del doble de la corriente inicial de 7.5 $\mathrm{A}$. El tiempo de establecimiento ante esta perturbación fue cercano a $5 \mathrm{~ms}$, y la señal de control se incrementa del $44 \%$ al $46 \%$.

Para validar la respuesta del controlador ante variaciones en el punto de operación debido a cambios en el voltaje de entrada del convertidor se modela un cambio de irradiación en el generador y un gradiente de temperatura, factores que afectan el rendimiento y la potencia generada por el panel solar. En la Figura 10 se observa una variación de irradiación de $1000 \mathrm{~W} / \mathrm{m}^{2}$ a $460 \mathrm{~W} / \mathrm{m}^{2}$ a los $15 \mathrm{~ms}$ y la respuesta del convertidor y el controlador, la cual mediante un incremento del ciclo de trabajo del $44 \%$ al $47 \%$, el cual compensa la variación del voltaje de salida del convertidor cuando el voltaje del generador fotovoltaico pasa de $122 V_{D C}$ a $116 V_{D C}$. 

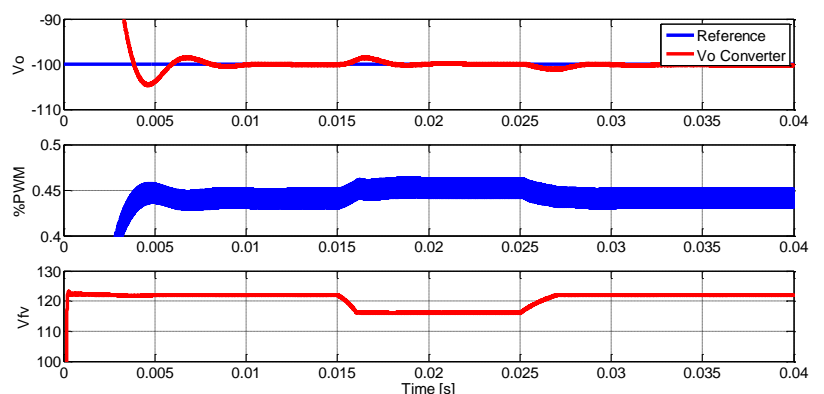

Figura 10. Respuesta transitoria ante variación de irradiación en el generador fotovoltaico.

La respuesta del controlador ante variación de temperatura en el generador fotovoltaico se muestra en la Figura 11. El panel solar se somete a una variación de $25^{\circ} \mathrm{C}$ a $70^{\circ} \mathrm{C}$, lo cual se traduce en una variación en el voltaje de salida del generador de $122 V_{D C}$ a $100 V_{D C}$. La señal de control para compensar esta variación se incrementó de $44 \%$ a $49 \%$, con un sobrepaso en la respuesta transitoria del $5 \%$ y un tiempo de establecimiento $7 \mathrm{~ms}$.
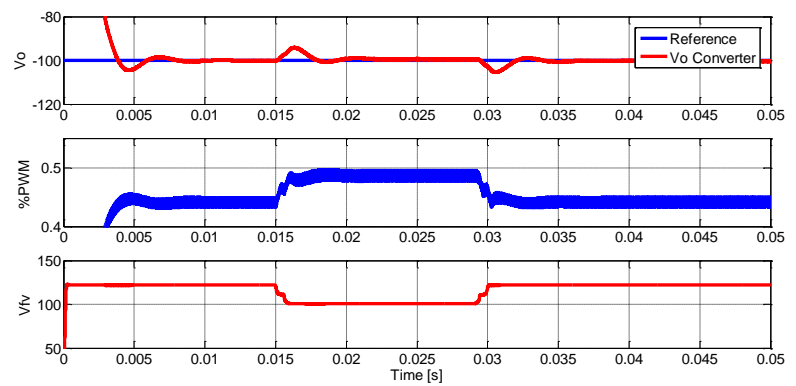

Figura 11. Respuesta transitoria ante variación de temperatura en el generador fotovoltaico.

\section{CONCLUSIONES}

El modelo linealizado en espacio de estado del convertidor es válido para el diseño del controlador QFT dado que describe adecuadamente la dinámica del circuito del convertidor, lo que permite el diseño de un controlador robusto que cumple las especificaciones de desempeño deseadas, para un esquema de control basado en el predictor Smith modificado para sistemas de fase no mínima, el cual permite sintonizar un controlador robusto QFT de dos grados de libertad dado que este esquema mitiga los efectos del cero en el semiplano derecho S.

El control robusto QFT para el regulador de tensión Buck-Boost aplicado a un generador fotovoltaico de $760 W p$ mostró un desempeño robusto ante las perturbaciones en la carga conectada al convertidor y variaciones en la tensión de entrada a causa de los cambios aleatorios de las condiciones ambientales (irradiación y temperatura) que se expone el panel solar en condiciones reales de funcionamiento, manteniendo un tiempo de establecimiento y sobrepaso acordes con las especificaciones de desempeño del controlador.

\section{REFERENCIAS}

[1] T. Kobaku, S. Patwardhan, and V. Agarwal, "Experimental Evaluation of Internal Model Control Scheme on a DC-DC Boost Converter Exhibiting Nonminimum Phase Behavior," IEEE Trans. Power Electron., vol. PP, no. 99, pp. 1-1, 2017.

[2] H. S. Khan and A. Y. Memon, "FIL simulation of sliding mode controller for DC/DC boost converter," in 2016 13th International Bhurban Conference on Applied Sciences and Technology (IBCAST), pp. 112-117, 2016.

[3] M. E. Şahın, H. İ. Okumuş, and H. Kahvecı, "Sliding mode control of PV powered DC/DC Buck-Boost converter with digital signal processor," in 2015 17th European Conference on Power Electronics and Applications (EPE'15 ECCE-Europe), pp. 1-8, 2015.

[4] A. Alam, L. Tao, K. Habib, and S. Khan, "Model predictive control for disturbance rejection and robust stability in Buck-Boost converter," in 2016 IEEE Advanced Information Management, Communicates, Electronic and Automation Control Conference (IMCEC), pp. 892-895, 2016.

[5] A. Alam, L. Tao, and K. Habib, "Optimal model predictive control for disturbance rejection and stability in buck-boost converter and its comparison with classical technique," in 2016 IEEE International Conference on Power System Technology (POWERCON), pp. 1-6, 2016.

[6] R. R. de C. Vaz, S. P. Pimentel, and S. Araújo, "Analysis and control of a non-inverter Buck-Boost power DC-DC converter by state-space modeling and applied to PV systems under MPPT operation," in 2015 IEEE PES Innovative Smart Grid Technologies Latin America (ISGT LATAM), pp. 338-343, 2015.

[7] M. Garcia-Sanz, Robust Control Engineering: Practical QFT Solutions. CRC Press, 2017.

[8] T. Khatib and W. Elmenreich, Modeling of Photovoltaic Systems Using MATLAB: Simplified Green Codes. John Wiley \& Sons, 2016.

[9] B. J. Saharia, M. Manas, and S. Sen, "Comparative Study on Buck and Buck-Boost DC-DC Converters for MPP Tracking for Photovoltaic Power Systems," in 2016 Second International Conference on Computational 
Intelligence Communication Technology (CICT), pp. 382-387, 2016.

[10]S. Bacha, I. Munteanu, and A. I. Bratcu, Power Electronic Converters Modeling and Control. London: Springer London, 2014.

[11]I. HOROWITZ and M. SIDI, "Optimum synthesis of non-minimum phase feedback systems with plant uncertainty†,," Int. J. Control, Apr. 2007.

[12]W.-H. Chen and D. J. Ballance, "QFT design for uncertain non-minimum phase and unstable plants revisited," Int. J. Control, vol. 74, no. 9, pp. 957-965, Enero 2001.

[13]M. Garcia-Sanz and C. H. Houpis, Wind Energy Systems: Control Engineering Design. CRC Press, 2012.

[14]A. A. Towati, Dynamic Analysis and QFT-based Robust Control Design of Switched-mode Power Converters. Helsinki University of Technology, 2008.

[15]M. G. Martínez, "Síntesis de controladores robustos mediante el análisis de la compatibilidad de especificaciones e incertidumbre," http://purl.org/dc/dcmitype/Text, Universidad Pública de Navarra, 2001.

[16]C. H. Houpis, S. N. Sheldon, and J. J. D'Azzo, Linear Control System Analysis and Design: Fifth Edition, Revised and Expanded. CRC Press, 2003.

[17]J. Elso, M. Gil-Martinez, and M. Garcia-Sanz, "Quantitative feedback control for multivariable model matching and disturbance rejection," Int. J. Robust Nonlinear Control, vol. 27, no. 1, pp. 121-134, Enero 2017.

[18]C. H. Houpis, S. J. Rasmussen, and M. Garcia-Sanz, Quantitative feedback theory: fundamentals and applications. CRC Press, 2005.

[19]M. Gil-Martínez and M. García-Sanz, "Simultaneous meeting of robust control specifications in QFT," Int. J. Robust Nonlinear Control, vol. 13, no. 7, pp. 643-656, 2003.

[20]Y. Chait and O. Yaniv, "Multi-input/single-output computer-aided control design using the quantitative feedback theory," Int. J. Robust Nonlinear Control, vol. 3, no. 1, pp. 47-54, Enero 1993. 